\title{
Expression and function of microRNA-27b in hepatocellular carcinoma
}

\author{
SHUJIE HE ${ }^{1}$, JINGYUAN ZHANG ${ }^{2}$, JUAN LIN ${ }^{3}$, CUISHENG ZHANG $^{4}$ and SHIJIE SUN ${ }^{4}$ \\ ${ }^{1}$ Department of Hepatobiliary Surgery, Yantai Mountain Hospital; ${ }^{2}$ Department of Medical Administration, \\ Yuhuangding Hospital; ${ }^{3}$ Department of Internal Medicine, Government Hospital of Yantai; \\ ${ }^{4}$ Department of Hepatobiliary Surgery, Yuhuangding Hospital, Yantai, Shandong 264001, P.R. China
}

Received February 22, 2015; Accepted December 11, 2015

DOI: $10.3892 / \mathrm{mmr} .2016 .4851$

\begin{abstract}
It has been reported that microRNAs (miRs) have key roles in tumorigenesis via inhibition of their target genes. Dysregulation of miR-27b has been detected in numerous types of human cancer, including hepatocellular carcinoma (HCC); however, the detailed role of miR-27b in HCC has yet to be elucidated. Reverse transcription-quantitative polymerase chain reaction and western blotting were performed to examine the mRNA and protein expression levels. Transwell assay and wound healing assay were used to determine cell invasion and migration. Luciferase reporter assay was to confirm the targeting relationship. The present study demonstrated that the expression levels of miR-27b were significantly increased in HCC cell lines, as compared with in normal human liver cells. In addition, miR-27b was frequently upregulated in HCC tissues, as compared with in normal adjacent tissues; the expression levels of miR-27b were increased in $77.1 \%$ (27/35) of the HCC tissue samples. Furthermore, elevated miR-27b expression levels were significantly correlated with tumor differentiation, Tumor Node Metastasis stage and vascular invasion $(\mathrm{P}<0.05)$. Knockdown of miR-27b expression inhibited HCC cell migration and invasion. Furthermore, Sprouty 2 (Spry2) was identified as a novel target of miR-27b in HCC HepG2 cells, and the protein expression levels of Spry2 were negatively regulated by miR-27b in HepG2 cells. Overexpression of Spry2 suppressed HCC cell migration and invasion, whereas downregulation of Spry2 reversed the suppressive effects of miR-27b inhibition on HCC cell migration and invasion. The results of the present study suggested that miR-27b may promote the migration and invasion of HCC cells, at least partially by suppressing Spry2 expression.
\end{abstract}

Correspondence to: Professor Shijie Sun, Department of Hepatobiliary Surgery, Yuhuangding Hospital, 20 Yuhuangding East Road, Yantai, Shandong 264001, P.R. China

E-mail: yantaisunshijie@163.com

Key words: hepatocellular carcinoma, microRNA-27b, Sprouty 2, clinicopathologic features, migration, invasion
Therefore, the miR-27b/Spry2 axis may be considered a potential therapeutic target for HCC.

\section{Introduction}

Hepatocellular carcinoma (HCC) is one of the most common types of malignancy in humans. There is an increasing incidence of HCC worldwide, and it is a leading cause of cancer-associated mortality, partly because many patients are initially diagnosed with advanced disease (1). In recent decades, great efforts have been made to determine the molecular mechanisms underlying HCC. In addition, dysregulated expression of oncogenes and tumor suppressors has been reported to have a key role in the development and progression of HCC (2).

Sprouty 2 (Spry2), which is a member of the sprouty family, is an evolutionarily conserved inhibitor of receptor tyrosine kinases (3). Spry2 has a suppressive role in the regulation of mitogen-activated protein kinase (MAPK) signaling (4). It has previously been reported that Spry2 is frequently downregulated in $\mathrm{HCC}$, and patients with lower Spry2 expression exhibit poorer survival and increased recurrence (5). Furthermore, downregulation of Spry2 is associated with highly malignant phenotypes, including advanced tumor stages and vascular invasion (5); however, the regulatory mechanism underlying Spry2 expression in HCC cells has yet to be elucidated.

MicroRNAs (miRs) are a class of 18-25 nucleotide long non-coding RNAs, which can induce mRNA degradation or suppress protein translation via binding to the 3'-untranslated regions (3'-UTRs) of specific mRNA (6). miRs are important post-transcriptional regulators of gene expression in normal cells, as well as in cancer cells. Therefore, assessment of miR levels could potentially be useful for the classification and stratification of tumors $(7,8)$. Dysregulated expression of specific miRs has been detected in HCC, including miR-122, miR-124, miR-138, miR-148a and miR-203 (9-12). Furthermore, upregulated miR-27b has been detected in five drug-resistant HCC cell lines (13). miR-27b has been suggested to serve as a possible marker of HCC following exposure to aflatoxins (14). However, the detailed role of miR-27b in mediating the metastatic potential of HCC cells remains unclear.

The present study aimed to investigate the association between miR-27b expression and HCC progression. In 
addition, the detailed role of miR-27b in the regulation of $\mathrm{HCC}$ cell migration and invasion was determined, and the underlying molecular mechanisms involving Spry2 were discussed.

\section{Materials and methods}

Reagents. Dulbecco's modified Eagle's medium (DMEM), fetal bovine serum (FBS), Lipofectamine 2000, TRIzol reagent and the miRNA Reverse Transcription kit were purchased from Thermo Fisher Scientific, Inc. (Waltham, MA, USA). miRNA quantitative polymerase chain reaction (qPCR) Detection kit was purchased from Genecopoeia (Rockville, MD, USA). Directed Mutagenesis kit was purchased from Agilent Technologies, Inc. (Santa Clara, CA, USA). Rabbit anti-human Spry2 monoclonal antibody (cat. no. ab180527), rabbit anti-human glyceraldehyde 3-phosphate dehydrogenase (GAPDH) monoclonal antibody (cat. no. ab8245) and mouse anti-rabbit secondary antibody IgG (HRP; cat. no. ab6728) were purchased from Abcam (Cambridge, MA, USA). Enhanced chemiluminescence (ECL) kit was purchased from Pierce Protein Biology; Thermo Fisher Scientific, Inc. (Rockford, IL, USA). PsiCHECK 2 vector was purchased from Promega Corporation (Madison, WI, USA). miR-27b mimics, miR-27b inhibitor, Spry2 plasmid and Spry2-specific small interfering (si)RNA were obtained from Guangzhou FulenGen Co., Ltd. (Guangzhou, China).

Tissue specimens. The present study was approved by the Ethics Committee of Yuhuangding Hospital (Yantai, China). A total of 35 primary HCC tissues and matched normal adjacent specimens were collected. Written informed consent was obtained. The histomorphology of all samples was confirmed by the Department of Pathology. HCC tissues were immediately snap-frozen in liquid nitrogen upon surgical removal.

Cell culture. The HepG2, LH86, LMH and PLHC-1 human HCC cell lines, and the THLE-3 normal liver cell line were obtained from the American Type Culture Collection (Manassas, VA, USA). The cells were cultured in DMEM supplemented with $10 \%$ fetal bovine serum (FBS) at $37^{\circ} \mathrm{C}$ in a humidified incubator containing $5 \% \mathrm{CO}_{2}$.

Reverse transcription-quantitative polymerase chain reaction (RT- $q P C R)$. Total RNA was extracted from tissues and cells using TRIzol reagent. Tissues were homogenized prior to RNA extraction by grinding. All samples were treated with $1 \mu \mathrm{l}$ DNase (Thermo Fisher Scientific, Inc.) prior to RT. The miRNA Reverse Transcription kit was used to reverse transcribe RNA into cDNA, according to the manufacturer's protocol. Briefly, reverse transcription was performed at $16^{\circ} \mathrm{C}$ for $30 \mathrm{~min}$, followed by an incubation step at $42^{\circ} \mathrm{C}$ for $30 \mathrm{~min}$ and enzyme inactivation at $85^{\circ} \mathrm{C}$ for $5 \mathrm{~min}$. qPCR was performed using a miRNA qPCR Detection kit on an ABI 7500 thermocycler (Applied Biosystems; Thermo Fisher Scientific, Inc., Waltham, MA, USA). The primers for Spry2 were: Forward: 5'-CCTACTGTCGTCCCAAGACCT-3' and reverse: 5'-GGGGCTCGTGCAGAAGAAT-3' purchased from Sangon Biotech Co., Ltd. (Shanghai, China). Briefly, the PCR cycling conditions were as follows: $50^{\circ} \mathrm{C}$ for $2 \mathrm{~min}$, $95^{\circ} \mathrm{C}$ for $10 \mathrm{~min}$, followed by 40 cycles of denaturation at $95^{\circ} \mathrm{C}$ for $15 \mathrm{sec}$ and annealing/elongation at $60^{\circ} \mathrm{C}$ for $60 \mathrm{sec}$. U6 was used as an internal reference gene. The experiment was repeated 3 times. The relative expression levels were analyzed using the $2^{-\Delta \Delta \mathrm{Cq}}$ method (15).

Western blotting. Tissues and cells were solubilized in cold radioimmunoprecipitation lysis buffer (Beyotime Biotechnology, Shanghai, China). Proteins were separated by $10 \%$ sodium dodecyl sulfate-polyacrylamide gel electrophoresis (50 $\mu \mathrm{g}$ of protein/lane), and transferred onto a polyvinylidene difluoride (PVDF; Thermo Fisher Scientific, Inc.) membrane. The membrane was incubated with phosphate-buffered saline containing 5\% milk overnight at $4^{\circ} \mathrm{C}$. Subsequently, the PVDF membrane was incubated with rabbit anti-Spry2 monoclonal antibody (1:50) and rabbit anti-GAPDH monoclonal antibody (1:50) at room temperature for $3 \mathrm{~h}$. The membrane was then incubated with mouse anti-rabbit secondary antibody $(1: 5,000)$ at room temperature for $1 \mathrm{~h}$. An ECL kit was used to perform chemiluminescent detection. The protein concentration was quantified using Pierce BCA Protein Assay Kit, Thermo Fisher Scientific, Inc. The relative protein expression levels were analyzed using Image-Pro Plus software 6.0 (Media Cybernetics, Inc., Rockville, MD, USA), and are presented as the density ratio vs. GAPDH.

Transfection. Lipofectamine 2000 was used to transfect the HepG2 cells, according to the manufacturer's protocol. Briefly, cells were cultured to $70 \%$ confluence, at a seed density of $10^{6}$ and resuspended in serum-free medium. miR-27b mimics, miR-27b inhibitor, Spry2 plasmid (Syngentech, Beijing, China), Spry2-specific siRNA and Lipofectamine 2000 were diluted with serum-free medium. The diluted Lipofectamine 2000 was added to the diluted siRNA and miR-27b mimics, and the mixture was incubated for $20 \mathrm{~min}$ at room temperature. Subsequently, the mixture was added to the cell suspension. After a $6 \mathrm{~h}$ incubation at $37^{\circ} \mathrm{C}$ and $5 \% \mathrm{CO}_{2}$, the medium was replaced with the normal serum-containing medium. Subsequently, the cells were cultured for $24 \mathrm{~h}$ prior to further experimentation.

Dual luciferase reporter assay. The miR-27b-predicted target sequence within the Spry2 3'-UTR (UUUGCAACU GUGAA), and a mutant lacking complimentarity with the miR-27b seed sequence (UUUGCACACACACC), were cloned downstream of the luciferase gene driven by the cytomegalovirus promoter, thus generating Luc.Spry2 and Luc.mtSpry2 vectors, respectively. The wild type (WT) and mutant (MUT) 3'UTRs of Spry2 were obtained from Biowit Technologies (Shenzhen, China), which was inserted into the $B g I I I$ and $K p n I$ restriction sites 3 ' to the end of the Renilla luciferase gene in the PsiCHECK vector. HepG2 cells were transfected with Luc.Spry2 or Luc.mtSpry2 vectors in conjunction with miR-27b mimics or scramble negative control miR mimics using Lipofectamine 2000. A total of $24 \mathrm{~h}$ post-transfection, the cells were harvested. Dual-Luciferase Reporter Assay System (Promega Corporation) was used to determine luciferase activity. Luciferase activity was quantified using an Lmax multiwell luminometer (Molecular Devices, Sunnyvale, CA, USA). 
Table I. Correlation between miR-27b expression and clinicopathologic features in patients with hepatocellular carcinoma.

\begin{tabular}{|c|c|c|c|c|c|}
\hline \multirow[b]{2}{*}{ Clinicopathologic feature } & \multirow[b]{2}{*}{ Cases } & \multicolumn{2}{|c|}{ miR-27b expression } & \multirow[b]{2}{*}{$\chi^{2}$} & \multirow[b]{2}{*}{ P-value } \\
\hline & & Low & High & & \\
\hline Age (years) & & & & 0.397 & 0.429 \\
\hline$<60$ & 12 & 2 & 10 & & \\
\hline$\geq 60$ & 23 & 6 & 17 & & \\
\hline Gender & & & & 0.027 & 0.602 \\
\hline Male & 21 & 5 & 16 & & \\
\hline Female & 14 & 3 & 11 & & \\
\hline Tumor size $(\mathrm{cm})$ & & & & 0.122 & 0.527 \\
\hline$<5$ & 15 & 3 & 12 & & \\
\hline$\geq 5$ & 20 & 5 & 15 & & \\
\hline Tumor differentiation & & & & 5.402 & 0.025 \\
\hline $\mathrm{I}+\mathrm{II}$ & 18 & 7 & 11 & & \\
\hline III+IV & 17 & 1 & 16 & & \\
\hline TNM stage & & & & 4.610 & 0.037 \\
\hline $\mathrm{I}+\mathrm{II}$ & 19 & 7 & 12 & & \\
\hline IIIA & 16 & 1 & 15 & & \\
\hline Vascular invasion & & & & 6.291 & 0.016 \\
\hline Yes & 17 & 7 & 10 & & \\
\hline No & 18 & 1 & 17 & & \\
\hline
\end{tabular}

TNM, Tumor Node Metastasis.

Wound healing assay. Wound healing assay was performed, in order to evaluate the cell migratory capacity of each group. Briefly, cells were cultured to full confluence. Wounds $\sim 1 \mathrm{~mm}$ wide were created in the cell layer using a plastic scriber, and cells were washed with phosphate-buffered saline and incubated in a serum-free medium. A total of $24 \mathrm{~h}$ after the wound was generated, cells were incubated in DMEM supplemented with $10 \%$ FBS. After further incubation for 0 and $48 \mathrm{~h}$, the cells were fixed with absolute alcohol and observed under a BX53 fluorescence microscope (Olympus Corporation, Tokyo, Japan).

Transwell assay. Transwell assay was performed in order to examine cell invasion. Briefly, the invasive ability of HepG2 cells was determined in 24-well Transwell chambers, which were coated with a layer of Matrigel. Cell suspension of DMEM $\left(10^{6}\right.$ cells $\left./ \mathrm{ml}\right)$ was added to the upper chamber, and DMEM supplemented with $10 \%$ FBS was added to the lower chamber. Following a $24 \mathrm{~h}$ incubation, non-invading cells as well as the Matrigel (Thermo Fisher Scientific, Inc.) on the interior of the inserts was removed using a cotton-tipped swab. Invasive cells on the lower surface of the membrane were stained with gentian violet, rinsed with water and air-dried. Five fields were randomly selected and cell number was counted under a microscope.

Bioinformatical analysis. The target genes of miR-27 were predicted using TargetScan (16). The following settings were used for analysis: i) Species, human; ii) gene symbol, Spry2 and iii) selelcted a conserved miRNA family.

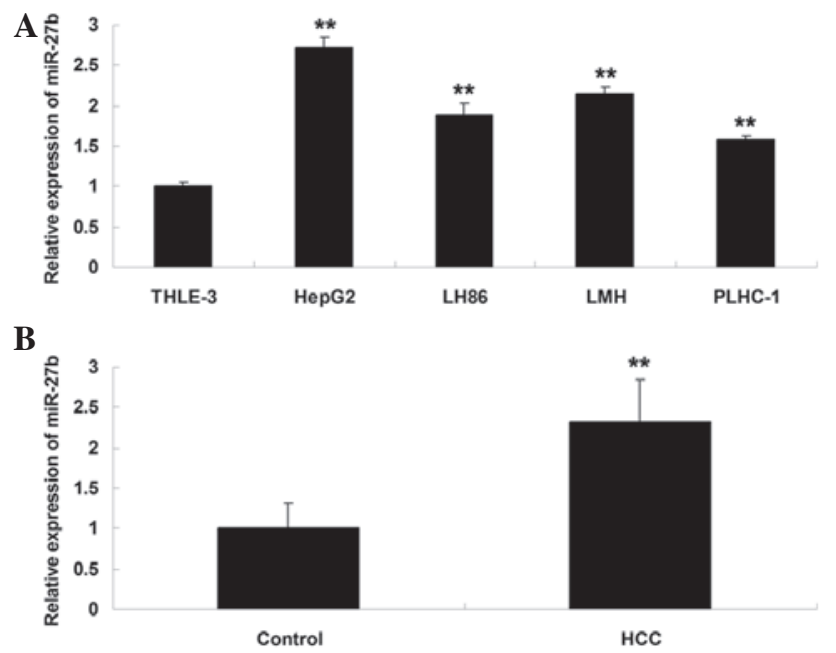

Figure 1. (A) Reverse transcription-quantitative polymerase chain reaction (RT-qPCR) was performed to determine the relative expression levels of microRNA (miR)-27b in human hepatocellular carcinoma (HCC) cell lines, HepG2, LH86, LMH and PLHC-1, as well as in a normal liver cell line, THLE-3. ${ }^{* *} \mathrm{P}<0.01$ vs. THLE-3 cells. (B) RT-qPCR was performed to determine the relative expression levels of miR-27b in HCC tissues and peritumoral noncancerous tissues (control). ${ }^{* *} \mathrm{P}<0.01$ vs. the control samples. Data are presented as the mean \pm standard deviation.

Statistical analysis. Data are presented as the mean \pm standard deviation. Statistical analysis was performed using SPSS 16.0 (SPSS, Inc., Chicago, IL, USA). The association between miR-27b expression and clinicopathologic features in patients 
A

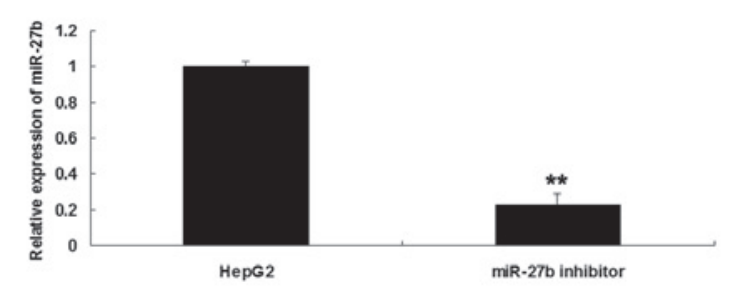

C
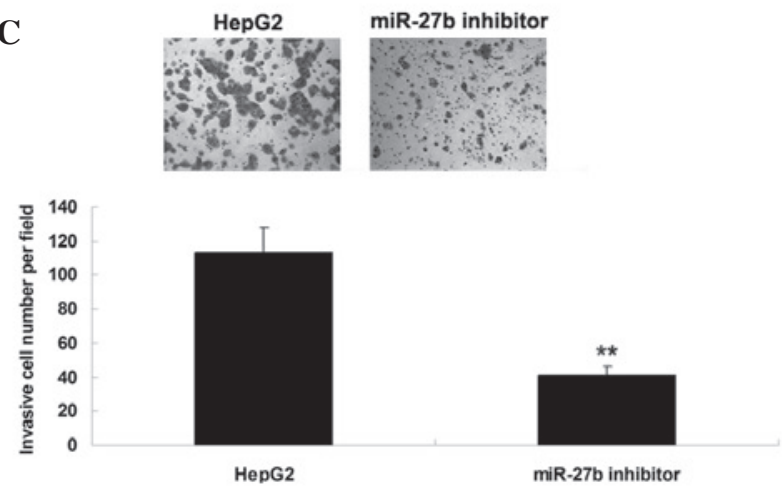

B
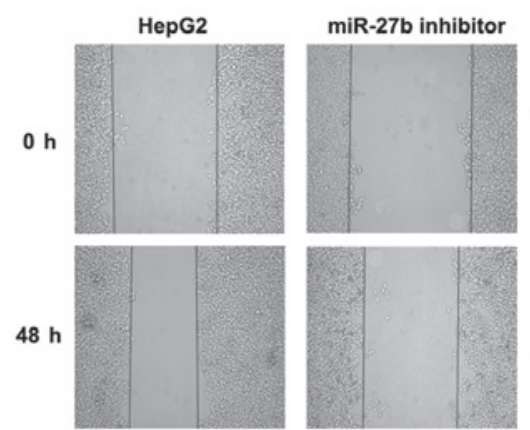
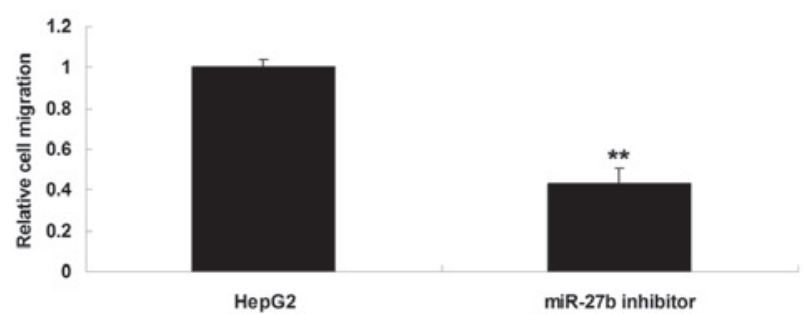

miR-27b inhibitor

Figure 2. (A) Reverse transcription-quantitative polymerase chain reaction was performed to determine the relative expression levels of miR-27b in HepG2 cells transfected with a microRNA (miR)-27b inhibitor. (B) Wound healing assay and (C) Transwell assay were performed to determine cell migration and invasion, respectively, in HepG2 cells transfected with miR-27b inhibitor. Untransfected HepG2 cells were used as a control. ${ }^{* *} \mathrm{P}<0.01$ vs. HepG2 cells. Data are presented as the mean \pm standard deviation.

with $\mathrm{HCC}$ was analyzed using $\chi^{2}$ test and Spearman rank correlation analysis. The statistical correlation of data between the cell groups was analyzed by one-way analysis of variance. $\mathrm{P}<0.05$ was considered to indicate a statistically significant difference.

\section{Results}

miR-27b expression pattern in HCC cell lines and tissue samples. The expression levels of miR-27b were examined in four common human HCC cell lines, HepG2, LH86, LMH and PLHC-1, as well as in a normal liver cell line, THLE-3. HepG2 cells exhibited a significant increase in the expression levels of miR-27b, as compared with the normal liver THLE-3 cells (Fig. 1A), therefore this cell line was used in further experiments. In addition, the expression levels of miR-27b were significantly increased in the HCC tissues, as compared with in the peritumoral noncancerous tissues (Fig. 1B). The expression levels of miR-27b were increased in $77.1 \%$ (27/35) of the HCC tissue samples.

Correlation between miR-27b expression and clinicopathologic features in patients with HCC. The association between miR-27b expression and clinicopathologic features in patients with HCC was analyzed using $\chi^{2}$ test. As presented in Table I, miR-27b expression had no association with the age, gender and tumor size of the patients $(\mathrm{P}>0.05)$. However, miR-27b expression was correlated with tumor differentiation, Tumor Node Metastasis (TNM) stage and vascular invasion $(\mathrm{P}<0.05)$.
Knockdown of miR-27b inhibits HepG2 cell migration and invasion. The present study investigated the effects of miR-27b on the regulation of migration and invasion in HCC cells. Since miR-27b was upregulated in HCC cells, HepG2 cells were transfected with a miR-27b inhibitor to suppress its expression. Post-transfection, the expression levels of miR-27b were reduced (Fig. 2A). Wound healing and Transwell assays were conducted to investigate the effects of miR-27b knockdown on HCC cell migration and invasion, respectively. As shown in Fig. 2B and C, knockdown of miR-27b expression significantly inhibited the migration and invasion of HepG2 cells. These results indicate that miR-27b has a role in the regulation of $\mathrm{HCC}$ metastasis.

Spry2 is identified as a target gene of miR-27b and is negatively regulated by miR-27b in HepG2 cells. Bioinformatical analysis was performed in order to predict the target genes of miR-27b, and Spry2 was identified as a putative target. To clarify whether Spry2 is a direct target of miR-27b, a Luc.Spry2 vector containing the wild-type 3'-UTR of Spry2 and a Luc.mtSpry 2 vector containing a mutated form of the 3'-UTR of Spry2 were generated (Fig. 3A). Subsequently, HepG2 cells were transfected with Luc.Spry2 or Luc.mtSpry2 vector, with or without miR-27b mimics. A total of $24 \mathrm{~h}$ post-transfection, the luciferase activity was examined. Compared with the control group, luciferase activity was significantly decreased in the HepG2 cells co-transfected with the Luc.Spry2 vector and miR-27b mimics, but was unaltered in HepG2 cells co-transfected with the Luc.mtSpry2 vector and miR-27b mimics (Fig. 3B). These results indicate 
A

$\begin{aligned} & \text { wild type of Spry2 3'-UTR } 5^{\prime} \text {-UUUGCAACUGUGAA-3' } \\ & \text { hI I I I I I I } \\ & \text { hsa-miR-27a } 5^{\prime} \text {-AAUCGGUGCACUU-3' } \\ & \text { mutant type of Spry2 } 3^{\prime} \text {-UTR } 5^{\prime} \text {-UUUGCACACACACC-3' }\end{aligned}$

C

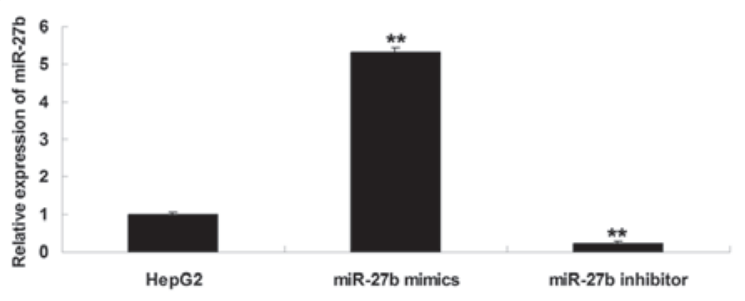

B

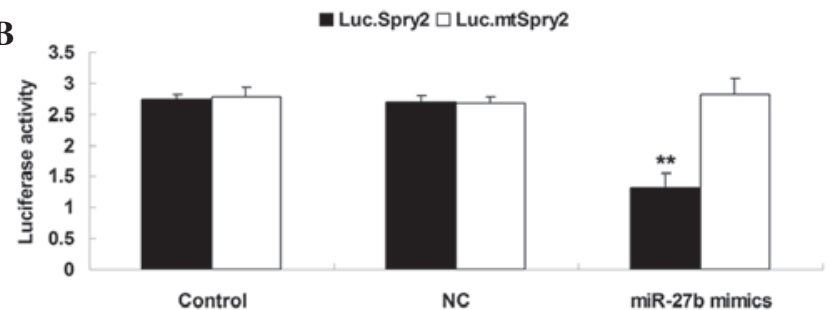

D
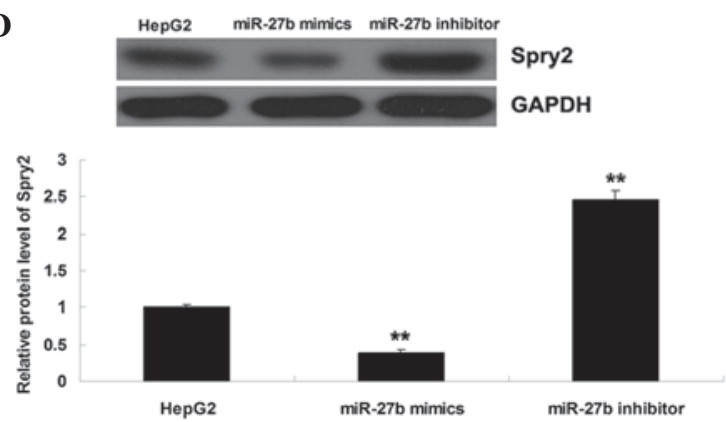

Figure 3. (A) Seed sequences of microRNA (miR)-27b in the wild-type or mutant type of Sprouty 2 (Spry2) 3'-untranslated region (UTR). (B) Luciferase activity was significantly decreased in HepG2 cells co-transfected with the Luc.Spry2 vector and miR-27b mimics, but was unaltered in HepG2 cells co-transfected with the Luc.mtSpry2 vector and miR-27b mimics, as compared with the control group. Control, cells transfected with Luc.Spry2 or Luc.mtSpry2 vectors only, without miR-27b mimics. Negative control (NC), cells transfected with Luc.Spry2 or Luc.mtSpry2 vectors, in conjunction with scramble miR mimics. ${ }^{* *} \mathrm{P}<0.01$ vs. the control cells. (C) Relative expression levels of miR-27b were detected in HepG2 cells transfected with miR-27b mimics or a miR-27b inhibitor. Untransfected HepG2 cells were used as a control. ${ }^{* *} \mathrm{P}<0.01$ vs. the HepG2 cells. (D) Protein expression levels of Spry2 were examined in HepG2 cells transfected with miR-27b mimics or a miR-27b inhibitor. Untransfected HepG2 cells were used as a control. ${ }^{* *} \mathrm{P}<0.01$ vs. the HepG2 cells. Data are presented as the mean \pm standard deviation. GAPDH, glyceraldehyde 3-phosphate dehydrogenase.

A
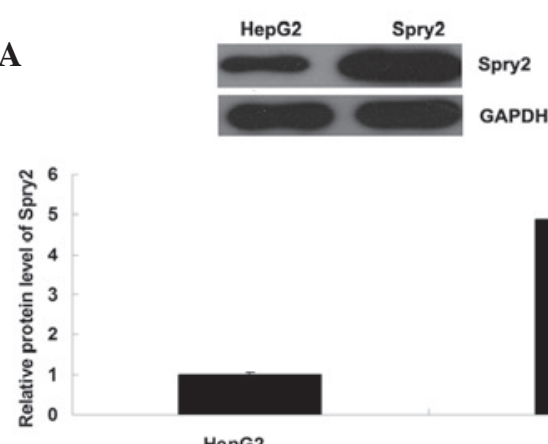

HepG2

C
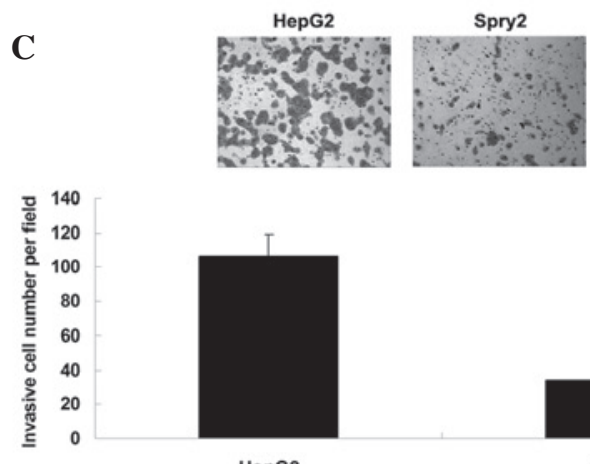

HepG2

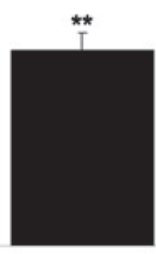

Spry2

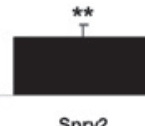

B

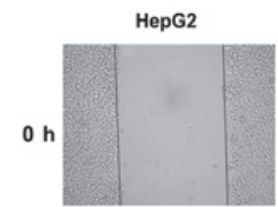

$48 \mathrm{~h}$
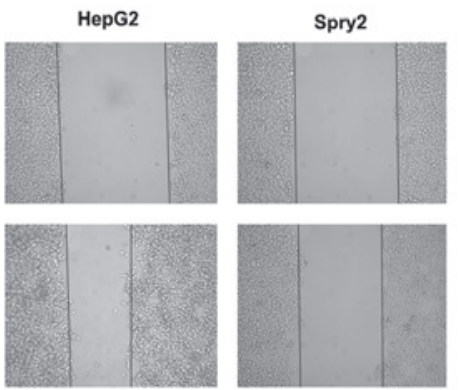

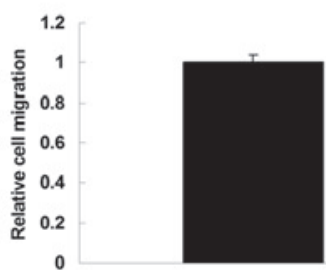

HepG2

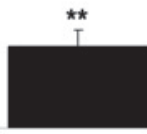

Spry2

Figure 4. (A) Protein expression levels of Sprouty 2 (Spry2) were detected in HepG2 cells transfected with Spry2 plasmid. (B) Wound healing and (C) Transwell assays were performed to determine cell migration and invasion, respectively, in HepG2 cells transfected with Spry2 plasmid. Untransfected HepG2 cells were used as a control. ${ }^{* *} \mathrm{P}<0.01$ vs. the HepG2 cells. Data are presented as the mean \pm standard deviation. GAPDH, glyceraldehyde 3-phosphate dehydrogenase.

that miR-27b directly binds to the seed sequences within the Spry2 3'-UTR in HepG2 cells.
The present study investigated the effects of miR-27b overexpression or knockdown on the protein expression 

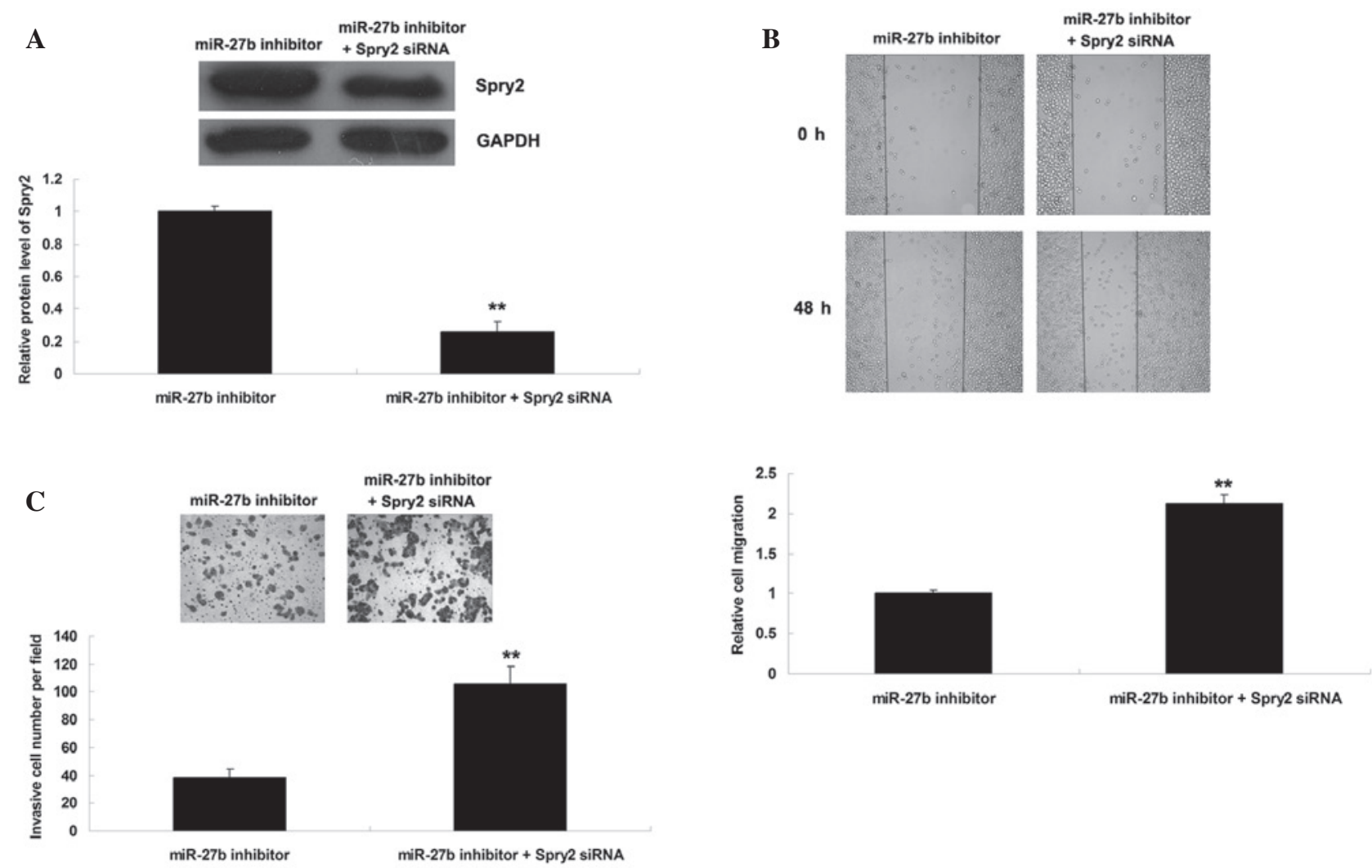

Figure 5. (A) Protein expression levels of Sprouty 2 (Spry2) were detected in HepG2 cells transfected with a microRNA (miR)-27b inhibitor, or co-transfected with a miR-27b inhibitor and Spry2 small interfering (si)RNA. (B) Wound healing and (C) Transwell assays were performed to determine cell migration and invasion, respectively, in HepG2 cells transfected with a miR-27b inhibitor, or co-transfected with miR-27b inhibitor and Spry2 siRNA. ${ }^{* *} \mathrm{P}<0.01 \mathrm{vs}$. cells transfected with a miR-27b inhibitor only. Data are presented as the mean \pm standard deviation. GAPDH, glyceraldehyde 3-phosphate dehydrogenase.

levels of Spry2 in HepG2 cells. HepG2 cells were transfected with miR-27b mimics or a miR-27b inhibitor. As shown in Fig. 3C, transfection with miR-27b mimics upregulated the miR-27b expression levels; however, transfection with the miR-27b inhibitor downregulated miR-27b expression levels. Subsequently, western blotting was performed to examine the protein expression levels of Spry2. As shown in Fig. 3D, upregulation of miR-27b significantly inhibited the protein expression levels of Spry2; however, knockdown of miR-27b expression enhanced the protein expression levels of Spry2 in HepG 2 cells. Accordingly, these results suggest that miR-27b may negatively regulate the protein expression levels of Spry2 in HCC HepG2 cells via directly targeting its mRNA.

Spry2 is involved in the miR-27b-mediated migration and invasion of HepG2 cells. Since knockdown of miR-27b upregulated Spry2 expression and suppressed HCC cell migration and invasion, the present study hypothesized that Spry 2 may be involved in miR-27b-mediated migration and invasion of HCC cells. To verify this hypothesis, HepG2 cells were transfected with a Spry2 plasmid. Post-transfection, the protein expression levels of Spry2 were significantly increased (Fig. 4A). Consistent with miR-27b knockdown, overexpression of Spry2 suppressed HCC cell migration and invasion (Fig. 4B and C).

To further clarify whether the effects of miR-27b on HCC cell migration and invasion were mediated by inhibition of Spry2 expression, HepG2 cells were transfected with a
miR-27b inhibitor, or co-transfected with a miR-27b inhibitor and Spry2 siRNA. Post-transfection, the protein expression levels of Spry2 were reduced in HepG2 cells co-transfected with a miR-27b inhibitor and Spry2 siRNA, as compared with the HepG2 cells transfected with a miR-27b inhibitor only (Fig. 5A). In addition, the migratory and invasive capacity was increased in HepG2 cells co-transfected with a miR-27b inhibitor and Spry2 siRNA, as compared with the HepG2 cells transfected with a miR-27b inhibitor only (Fig. 5B and C). These results indicate that knockdown of Spry2 expression may reverse the suppressive effects of miR-27b inhibition on HCC cell migration and invasion. Based on these findings, miR-27b may have a promoting role in the regulation of HCC cell migration and invasion, at least partially via directly targeting Spry2 expression.

\section{Discussion}

Dysregulated expression of miR-27b has previously been implicated in HCC (13); however, the detailed role of miR-27b in HCC remains largely unknown. The results of the present study demonstrated that the expression levels of miR-27b were increased in HCC cell lines and tissues samples. Furthermore, elevated miR-27b expression was significantly correlated with histological grade, depth of invasion and TNM stage. Further investigation revealed that miR-27b had a promoting role in the regulation of HCC cell migration and invasion, at least partially via directly targeting Spry2 expression. 
Dysregulated miR expression has been shown to be tightly associated with the development and progression of $\operatorname{HCC}(1,17)$. Dysregulation of miR-148a discriminates not only the overall survival and recurrence free survival rates of $\mathrm{HCC}$, but also the vascular invasion (18). miR-27b has previously been reported to be involved in hepatic lipid metabolism (19). In addition, miR-27b has been suggested to be involved in $\operatorname{HCC}(13,14)$. Zhuo et al (13) investigated the association between miRs and multidrug resistance using the HCC Huh-7 cell line, which was treated with adramycin, cisplatin, carboplatin, mitomycin $\mathrm{C}$ or vincristine at increasing concentrations, in order to develop drug-resistant sublines. miR-27b was significantly upregulated in the drug-resistant HCC cell lines, suggesting that miR-27b may have a role in drug resistance in HCC (13). Furthermore, miR-27b has been suggested to serve as a possible marker of HCC following exposure to aflatoxins (14). The present study is the first, to the best of our knowledge, to demonstrate that miR-27b was frequently upregulated in HCC, and elevated miR-27b expression levels were significantly correlated with tumor differentiation, TNM stage and vascular invasion. These findings suggested that miR-27b may act as an oncogenic miR in HCC. Further studies should expand the sample size, and focus on the association between miR-27b expression and the prognosis of patients with HCC.

The role of miR-27b has also been demonstrated in other types of cancer, with the majority of studies suggesting that miR-27b may act as a tumor suppressor in human cancer $(20,21)$. Lee et al (22) demonstrated that miR-27b inhibited growth, progression and the inflammatory response in neuroblastoma cells by targeting peroxisome proliferator-activated receptor $\gamma$. In addition, miR-27b was downregulated in non-small cell lung cancer (NSCLC), and overexpression of miR-27b significantly suppressed the proliferation and invasion of NSCLC cells (21). However, in several types of human malignancy, miR-27b acts as an oncogene (23). miR-27b was upregulated in glioma tissues and cells, and knockdown of miR-27b was able to trigger growth inhibition, induce apoptosis and inhibit invasion of glioma cells, potentially via direct or indirect inhibition of signal transducer and activator of transcription 3 , c-myc and cyclin D1 (24). The present study demonstrated that miR-27b had an oncogenic role in the regulation of HCC cell migration and invasion.

Since miRs have roles in human cancer via mediating the protein expression of their target genes (25), the present study focused on the target genes of miR-27b in HCC cells. Spry2 was identified as a direct target gene of miR-27b in HCC cells. A previous study reported that dysregulated Spry2 expression has been detected in HCC (5). Song et al (5) demonstrated that $86.3 \%$ (207 of 240) of patients with HCC exhibited downregulated Spry2 expression. Patients negative for Spry2 exhibited poorer survival and increased recurrence. Multivariate analysis further established Spry2 as an independent predictor of postoperative recurrence in patients with HCC. Furthermore, downregulation of Spry2 was associated with highly malignant phenotypes, and was positively correlated with the metastatic potential of HCC cell lines (5). Lee et al (26) investigated the potential role of Spry 2 in HCC by expressing dominant negative Spry 2 and activated $\beta$-catenin in the mouse liver; the results demonstrated that tumor cells exhibited high expression levels of extracellular signal-regulated kinases, as well as dysregulation of genes associated with cell proliferation, apoptosis and angiogenesis. In addition, Wang et al (27) reported that inactivation of Spry2 enhanced AKT-driven hepatocarcinogenesis via activation of MAPK and pyruvate kinase muscle pathways. The present study demonstrated that overexpression of Spry2 suppressed HCC cell migration and invasion, suggesting that Spry2 has a suppressive role in HCC metastasis. Furthermore, knockdown of Spry2 reversed the suppressive effects of miR-27b inhibition on HCC cell migration and invasion, thus suggesting that Spry 2 is involved in miR-27b-meditated HCC cell migration and invasion.

In conclusion, the present study demonstrated that upregulation of miR-27b may be associated with HCC progression, and suggested that miR-27b promotes the migration and invasion of HCC cells, at least partially via suppressing Spry2 expression. Therefore, the miR-27b/Spry2 axis may be considered a potential therapeutic target for HCC.

\section{References}

1. Zhu AX: Molecularly targeted therapy for advanced hepatocellular carcinoma in 2012: Current status and future perspectives. Semin Oncol 39: 493-502, 2012.

2. Psyrri A, Arkadopoulos N, Vassilakopoulou M, Smyrniotis V and Dimitriadis G: Pathways and targets in hepatocellular carcinoma. Expert Rev Anticancer Ther 12: 1347-1357, 2012.

3. Li P, Tao L, Yang J, Cai H, Ju X, Li J, Shao P, Cao Q, Qin C, Meng $\mathrm{X}$ and Yin C: Sprouty2 is associated with prognosis and suppresses cell proliferation and invasion in renal cell carcinoma. Urology 82: 253. e1-e7, 2013.

4. Mei Y, Bian C, Li J, Du Z, Zhou H, Yang Z and Zhao RC: miR-21 modulates the ERK-MAPK signaling pathway by regulating SPRY2 expression during human mesenchymal stem cell differentiation. J Cell Biochem 114: 1374-1384, 2013.

5. Song K, Gao Q, Zhou J, Qiu SJ, Huang XW, Wang XY and Fan J: Prognostic significance and clinical relevance of Sprouty 2 protein expression in human hepatocellular carcinoma. Hepatobiliary Pancreat Dis Int 11: 177-184, 2012.

6. Ambros V: The functions of animal microRNAs. Nature 431: 350-355, 2004.

7. Bartel DP: MicroRNAs: Genomics, biogenesis, mechanism, and function. Cell 116: 281-297, 2004.

8. Calin GA and Croce CM: MicroRNA signatures in human cancers. Nat Rev Cancer 6: 857-866, 2006.

9. Coulouarn C, Factor VM, Andersen JB, Durkin ME and Thorgeirsson SS: Loss of miR-122 expression in liver cancer correlates with suppression of the hepatic phenotype and gain of metastatic properties. Oncogene 28: 3526-3536, 2009.

10. Furuta M, Kozaki KI, Tanaka S, Arii S, Imoto I and Inazawa J: miR-124 and miR-203 are epigenetically silenced tumor-suppressive microRNAs in hepatocellular carcinoma. Carcinogenesis 31: 766-776, 2010.

11. Wang W, Zhao LJ, Tan YX, Ren H and Qi ZT: MiR-138 induces cell cycle arrest by targeting cyclin D3 in hepatocellular carcinoma. Carcinogenesis 33: 1113-1120, 2012.

12. Yin W, Zhao Y, Ji YJ, Tong LP, Liu Y, He SX and Wang AQ: Serum/plasma microRNAs as biomarkers for HBV-related hepatocellular carcinoma in China. Biomed Res Int 2015: 965185, 2015.

13. Zhuo L, Liu J, Wang B, Gao M and Huang A: Differential miRNA expression profiles in hepatocellular carcinoma cells and drug-resistant sublines. Oncol Rep 29: 555-562, 2013.

14. Valencia-Quintana R, Sánchez-Alarcón J, Tenorio-Arvide MG, Deng Y, Montiel-González JM, Gómez-Arroyo S, Villalobos-Pietrini R, Cortés-Eslava J, Flores-Márquez AR and Arenas-Huertero F: The microRNAs as potential biomarkers for predicting the onset of aflatoxin exposure in human beings: A review. Front Microbiol 5: 102 , 2014.

15. Livak KJ and Schmittgen TD: Analysis of relative gene expression data using real-time quantitative PCR and the 2(-Delta Delta C(T)) Method. Methods 25: 402-408, 2001. 
16. Lewis BP, Burge CB and Bartel DP: Conserved seed pairing, often flanked by adenosines, indicates that thousands of human genes are microRNA targets. Cell 120:15-20, 2005.

17. Zhang Y, Guo X, Xiong L, Kong X, Xu Y, Liu C, Zou L, Li Z, Zhao J and Lin N: MicroRNA-101 suppresses SOX9-dependent tumorigenicity and promotes favorable prognosis of human hepatocellular carcinoma. FEBS Lett 586: 4362-4370, 2012.

18. Heo MJ, Kim YM, Koo JH, Yang YM, An J, Lee SK, Lee SJ, Kim KM, Park JW and Kim SG: microRNA-148a dysregulation discriminates poor prognosis of hepatocellular carcinoma in association with USP4 overexpression. Oncotarget 5: 2792-2806, 2014.

19. Her GM, Hsu CC, Hong JR, Lai CY, Hsu MC, Pang HW, Chan SK and Pai WY: Overexpression of gankyrin induces liver steatosis in zebrafish (Danio rerio). Biochim Biophys Acta 1811: 536-548, 2011.

20. Ishteiwy RA, Ward TM, Dykxhoorn DM and Burnstein KL: The microRNA -23b/-27b cluster suppresses the metastatic phenotype of castration-resistant prostate cancer cells. PLoS One 7: e52106, 2012.

21. Wan L, Zhang L, Fan K and Wang J: MiR-27b targets LIMK1 to inhibit growth and invasion of NSCLC cells. Mol Cell Biochem 390: 85-91, 2014.
22. Lee JJ, Drakaki A, Iliopoulos D and Struhl K: MiR-27b targets PPAR $\gamma$ to inhibit growth, tumor progression and the inflammatory response in neuroblastoma cells. Oncogene 31: 3818-3825, 2012.

23. Jin L, Wessely O, Marcusson EG, Ivan C, Calin GA and Alahari SK: Prooncogenic factors miR-23b- and miR-27b are regulated by Her2/Neu, EGF, and TNF- $\alpha$ in breast cancer. Cancer Res 73: 2884-2896, 2013.

24. Chen L, Li H, Han L, Zhang K, Wang G, Wang Y, Liu Y, Zheng Y, Jiang T, Pu P, et al: Expression and function of miR-27b in human glioma. Oncol Rep 26: 1617-1621, 2011.

25. Yoshitaka T, Kawai A, Miyaki S, Numoto K, Kikuta K, Ozaki T, Lotz $\mathrm{M}$ and Asahara $\mathrm{H}$ : Analysis of microRNAs expressions in chondrosarcoma. J Orthop Res 31: 1992-1998, 2013.

26. Lee SA, Ho C, Roy R, Kosinski C, Patil MA, Tward AD, Fridlyand $\mathrm{J}$ and Chen $\mathrm{X}$ : Integration of genomic analysis and in vivo transfection to identify sprouty 2 as a candidate tumor suppressor in liver cancer. Hepatology 47: 1200-1210, 2008.

27. Wang C, Delogu S, Ho C, Lee SA, Gui B, Jiang L, Ladu S, Cigliano A, Dombrowski F, Evert M, et al: Inactivation of Spry2 accelerates AKT-driven hepatocarcinogenesis via activation of MAPK and PKM2 pathways. J Hepatol 57: 577-583, 2012. 